\title{
Review: allylamines, azoles, undecenoic acid, and tolnaftate are effective for fungal skin infections of the foot
}

Crawford F, Hart R, Bell-Syer S, et al. Topical treatments for fungal infections of the skin and nails of the foot. (Cochrane Review, latest version 10 May 1999). In: Cochrane Library. Oxford: Update Software.

QUESTION: Are topical treatments effective for fungal infections of the skin and nails of the foot?

\section{Data sources}

Studies were identified using Medline (1966 to December 1997), EMBASE/Excerpta Medica (1980 to December 1997), CINAHL (inception to December 1997), Cochrane Controlled Trials Register, Science and Social Science Citation Indexes, CAB-Health, Healthstar, DARE, NHS Economic Evaluation, and EconLit and by scanning bibliographies of relevant and unpublished studies, handsearching journals, and contacting drug companies and experts.

\section{Study selection}

Randomised controlled trials were selected if they evaluated topical treatments for mycologically diagnosed fungal infections of the skin and nails of the foot.

\section{Data extraction}

Study methodology and quality, participant characteristics, intervention regimens and costs, mycological cure or recurrence, quality of life, and adverse events.

\section{Main results}

126 trials were identified and 72 (70 of treatment of skin infections of the foot and 2 of treatment of nail infections of the foot) met the inclusion criteria. Allylamines, azoles, undecenoic acid, and tolnaftate had lower failure to cure rates than placebo for fungal skin infections of the foot (table). Compared with azoles, allylamines had lower failure to cure rates for fungal skin infections (relative risk 88\%, 95\% CI 78\% to 99\%). 2 trials of topical treatments compared with placebo showed no difference in failure to cure rates for fungal nail infections. and Development for Health and Social Care, UK.

For correspondence: Ms F Crawford,

Department of Health

Sciences and Clinical

Evaluation, The

University of York, Alcuin

College, Heslington, York

YO1 5DD, UK. Fax +44

(0) 1904434503

\section{Conclusion}

Allylamines, azoles, undecenoic acid, and tolnaftate are more effective than no treatment and allylamines are more effective than azoles for fungal skin infections of the foot.

\section{COMMENTARY}

Much has been written on the treatment of common fungal infections of the skin of the foot (eg, athlete's foot). Few studies, however, have compared the outcomes of cure rate, cost, or client quality of life for topical products on the market. Even fewer studies have compared topical treatments of fungal nail infection. The meta-analysis by Crawford $e t$ al compared the effects of topical products for fungal infections of the skin and nails of the foot on cure rates and cost to make recommendations about the clinical application of the results.

Studies included in the meta-analysis were identified by a thorough search of published and unpublished literature from English and non-English sources. Although it is not clear how data were extracted from non-English manuscripts, their inclusion strengthened the study. The mean number of quality criteria met by the 72 included trials was only 6.3 out of 12 . This low mean may suggest weaknesses in the studies and reduce confidence in the conclusions of the individual trials.

Despite the weaknesses identified above, this review provides useful information for a variety of providers Telephone triage nurses, school nurses, or nurse practitioners responding to a complaint of itchy, burning, peeling skin on and around the toes and soles of the foot may use the findings of this study to guide recommendations for initial selection of a topical treatment.

The authors conclude that the allylamines were slightly more effective than the azoles, but were much more expensive. Providers treating this non-life threatening, persistent, and uncomfortable problem are advised that the selection of azoles or undecenoic acid is the most cost effective strategy for first line treatment of fungal infections of the skin of the foot. The availability of some of the topical treatments varies by country-for example, undecenoic acid is only available in the USA as a multi-ingredient cream (eg, "Phicon F" and "Dermasept antifungal", both which also contain tolnaftate). In the UK, undecenoic acid is available in a single ingredient preparation (eg, Mycota).

Few studies were identified that evaluated topical treatments for fungal nail infections and, therefore, it is not possible to make recommendations on the use of topical treatments for this problem.

Mary Jo Gagan, RNCS, FNP, PhD Assistant Professor, Nurse Practitioner Options College of Nursing, University of Arizona Tucson, Arizona, USA

Comparison of topical treatment regimens for failure to cure fungal skin infections of the foot at $1-8$ weeks of follow up*

\begin{tabular}{llll}
$\begin{array}{l}\text { Comparison regimens } \\
\text { (weighted event rates) }\end{array}$ & $\begin{array}{l}\text { Number of } \\
\text { studies }\end{array}$ & RRR (95\% CI) & NNT (CI) \\
\hline Allylamines $(23 \%) v$ placebo $(80 \%)$ & 12 & $65 \%(56$ to 72$)$ & $2(2$ to 3$)$ \\
\hline Azoles $(23 \%) v$ placebo $(61 \%)$ & 17 & $68 \%(56$ to 76$)$ & $3(3$ to 4$)$ \\
\hline Undecenoic acid $(29 \%) v$ placebo $(75 \%)$ & 4 & $61 \%(44$ to 72$)$ & $3(2$ to 4$)$ \\
\hline Tolnaftate $(52 \%) v$ placebo $(70 \%)$ & 3 & $26 \%(4$ to 43$)$ & $6(4$ to 31$)$ \\
\hline
\end{tabular}

*Abbreviations defined in glossary; RRR, NNT, and Cl calculated from data provided by the author. 